\title{
VARIETIES AND UNIVERSAL MODELS IN THE THEORY OF COMBINATORIAL GEOMETRIES
}

\author{
BY JEFF KAHN AND JOSEPH P. S. KUNG
}

One of the most attractive but little studied ideas in the theory of combinatorial geometries (or matroids) [2], [4] is the notion of a hereditary class of geometries. A hereditary class of (finite) geometries is a collection of geometries which is closed under taking minors and direct sums. Thus, hereditary classes are direct analogues of varieties in universal algebra. Although varieties are highly structured collections of objects-free objects exist, for example-hereditary classes are relatively unstructured. In order to obtain reasonable results, it is necessary to impose some regularity conditions. One possibility is to postulate the existence of free (or rather, cofree) objects. A sequence of universal models for a hereditary class $T$ of geometries is a sequence $\left(T_{n}\right)$ of geometries in $T$ with $\operatorname{rank}\left(T_{n}\right)=$ $n$ satisfying the universal property: if $G$ is a geometry in $T$ of rank $n$, then $G$ is a subgeometry of $T_{n}$. A variety of geometries is a hereditary class with a sequence of universal models. Rather surprisingly, it is possible to classify varieties of geometries.

Before stating our result, we need to describe two simple sequences of geometries. Let $M_{1}$ be the rank one geometry and $M_{2}$ be the line with $q+1$ points. Let $M_{2 n}(q)=M_{2} \oplus \cdots \oplus M_{2}$ and $M_{2 n+1}(q)=M_{2} \oplus \cdots \oplus M_{2} \oplus M_{1}$. The subgeometries of these geometries form a variety called the variety of matchstick geometries of order $q$. Now, let $B_{n}$ be the Boolean algebra on the set $\{1, \ldots, n\}$. On each of the lines $\overline{12}, \overline{23}, \ldots, \overline{(n-1) n}$, add $q-1$ points in general position and call the resulting geometry $O_{n}(q)$. The subgeometries of these geometries also form a variety called the variety of origami geometries of order $q$.

THEOREM. Let $T$ be a variety of geometries. Then, $T$ is one of the following collections:

1. the variety of free geometries;

2. the variety of matchstick geometries of order $q$;

3. the variety of origami geometries of order $q$;

4. the variety of all geometries coordinatizable over a fixed finite field $G F(q)$;

5. the variety of voltage-graphic geometries with voltages in a fixed finite group $A$.

Received by the editors March 5, 1980.

AMS (MOS) subject classifications (1970). Primary 05B35, 08A15.

() 1980 American Mathematical Society 0002-9904/80/0000-0405/\$01.50 
The last entry merits some description. The universal models for the $A$ voltage-graphic geometries are the Dowling geometries $Q_{n}(A)$ [3]. Voltage-graphic geometries were discovered by Zaslavsky and their properties may be found in $[5],[6]$.

We conclude with an outline of the proof. Suppose that $\left(T_{n}\right)$ is the sequence of universal models for $T$ and let $q$ be the positive integer $\left|T_{2}\right|-1$. If $q=1$, then $T_{n}$ is a Boolean algebra. Assume that $q \geqslant 2$. If $T_{3}$ is not connected, then $T_{n}$ is the full matchstick geometry $M_{n}(q)$. We assume $T_{3}$ is connected and distinguish two cases: (a) for some $n, T_{n}$ splits: that is to say, $T_{n}$ is the union of two proper flats; (b) no $T_{n}$ splits.

Case (a). Let $m$ be the smallest index for which $T_{m+1}$ splits. We first show that for $n \geqslant m, T_{n}$ is the union of $n-m+1$ flats isomorphic to $T_{m}$, and obtain a fairly precise description of how these flats are arranged in $T_{n}$. By a counting argument we show that $m=2$. It follows that $T_{n}$ is the origami geometry $O_{n}(q)$.

Case (b). $T_{3}$ is shown to contain three modular lines $l_{1}, l_{2}, l_{3}$ in general position. If $T_{3}$ in the union of these lines, then $T_{n}$ is shown to be isomorphic to $Q_{n}(A)$ for some finite group $A$. The key idea here is to use contractions in $T_{4}$ to prove associativity in the quasigroup $A$ defined by $T_{3}$ (see [3]). If $T_{3}$ is not the union of $l_{1}, l_{2}, l_{3}$, then we again use contractions in $T_{4}$, this time to prove that $T_{3}$ is a projective plane. An inductive argument then shows that $T_{n}$ is the projective geometry $P_{n}(q)$ of rank $n$ over $G F(q)$.

\section{REFERENCES}

1. P. M. Cohn, Universal algebra, Harper and Row, New York, 1965.

2. H. H. Crapo and G.-C. Rota, On the foundations of combinatorial theory. II. Combinatorial geometries (preliminary ed.), M.I.T. Press, Cambridge, Mass., 1970.

3. T. A. Dowling, $A$ class of geometric lattices based on finite groups, J. Combinatorial Theory (B) 14 (1973), 61-86.

4. D. J. A. Welsh, Matroid theory, Academic Press, London, 1976.

5. T. Zaslavsky, Biased graphs, 1977 (preprint).

6. $\longrightarrow$, Signed graphs, 1978 (preprint).

DEPARTMENT OF MATHEMATICS, MASSACHUSETTS INSTITUTE OF TECHNOLOGY, CAMBRIDGE, MASSACHUSETTS 02139

Current address (Joseph Kung): Department of Mathematics, North Texas State University, Denton, Texas 76203 